\title{
Análisis de concordancia de tres clasificaciones de terceros molares mandibulares retenidos
}

\author{
Robert Stick Rivera-Herrera, ${ }^{1}$ Vicente Esparza-Villalpando, ${ }^{2}$ Josué Roberto Bermeo-Escalona, ${ }^{3}$ \\ Ricardo Martínez-Rider' y Amaury Pozos-Guillén * * \\ ${ }^{1}$ Universidad Autónoma de San Luis Potosí, Facultad de Estomatología, San Luis Potosí; ' Universidad Autónoma de San Luis Potosí, Ingeniería \\ y Ciencia de Materiales, San Luis Potosí; ${ }^{3}$ Universidad De La Salle Bajío, Facultad de Odontología, Guanajuato. México
}

\section{Resumen}

Introducción: Las clasificaciones de Pell y Gregory y de Winter son básicas en la categorización de terceros molares; la clasificación de Sánchez Torres es usada en México, pero no había sido evaluada previamente. Objetivo: Evaluar el grado de acuerdo en la valoración radiográfica de terceros molares mandibulares impactados, con el empleo de tres clasificaciones: Pell y Gregory, Winter y Sánchez Torres. Método: Estudio observacional, descriptivo, de concordancia interobservador, que incluyó a 10 cirujanos orales y maxilofaciales y 10 residentes en formación, quienes registraron la categorización radiográfica de terceros molares mandibulares (izquierdos y derechos) de acuerdo con las clasificaciones de Pell y Gregory, Sánchez Torres y Winter. Se evaluó el grado de acuerdo entre observadores mediante la prueba de kappa de Fleiss. Resultados: La clasificación de Pell y Gregory obtuvo el menor grado de acuerdo (kappa = 0.05 y 0.185), seguida de la clasificación de Sánchez Torres (kappa = 0.125 y 0.326); el mejor valor lo obtuvo la clasificación de Winter, con kappa =0.28 y 0.636 para cirujanos orales y maxilofaciales y residentes en formación, respectivamente. Conclusión: La clasificación de Winter mostró un grado de acuerdo aceptable (moderado) para categorizar terceros molares mandibulares en los residentes en formación.

PALABRAS CLAVE: Tercer molar. Exéresis dental. Clasificación de Pell y Gregory. Clasificación de Winter. Clasificación de Sánchez-Torres.

\section{Agreement analysis of three mandibular third molar retention classifications}

\begin{abstract}
Introduction: Pell \& Gregory and Winter classifications are basic in third molar categorization; Sánchez-Torres classification is used in Mexico, but it has not been previously evaluated. Objective: To assess the degree of agreement in the radiographic evaluation of impacted mandibular third molar with the use of three classifications: Pell \& Gregory, Winter and SánchezTorres. Method: Observational, descriptive, inter-observer degree of agreement study that included 10 oral and maxillofacial surgeons and 10 training residents, who recorded the radiographic categorization of third mandibular molars (left and right) according to Pell and Gregory, Sánchez-Torres and Winter classifications. Inter-observer degree of agreement was assessed with Fleiss' kappa test. Results: Pell and Gregory classification obtained the lowest degree of agreement (kappa $=0.05$ and 0.185), followed by Sánchez-Torres classification (kappa = 0.125 and 0.326); Winter had the best agreement, with kappa = 0.28 and 0.636 for oral and maxillofacial surgeons and training residents, respectively. Conclusion: The Winter classification showed an acceptable (moderate) degree of agreement to classify mandibular third molars by training residents.
\end{abstract}

KEY WORDS: Third molar. Exeresis of retained teeth. Pell and Gregory classification. Winter classification. Sánchez-Torres classification.

Correspondencia:

*Amaury Pozos-Guillén

E-mail: apozos@uasip.mx
Fecha de recepción: 03-03-2019

Fecha de aceptación: 30-05-2019

DOI: $10.24875 /$ GMM. 19005113
Gac Med Mex. 2020;156:22-26

Disponible en PubMed

www.gacetamedicademexico.com

0016-3813/@ 2019 Academia Nacional de Medicina de México, A.C. Publicado por Permanyer. Éste es un artículo open access bajo la licencia CC BY-NC-ND (http://creativecommons.org/licenses/by-nc-nd/4.0/). 


\section{Introducción}

La exéresis de los órganos dentarios retenidos es una de las prácticas más recurrentes en la cirugía oral y maxilofacial, ${ }^{1}$ especialmente de los terceros molares mandibulares, uno de los órganos dentales con mayor frecuencia de impactación. ${ }^{2}$ Las indicaciones para la remoción dentaria son variadas y están relacionadas con la posición, forma y patologías asociadas con cada órgano. ${ }^{3}$ Las características anatómicas de los terceros molares incluyen coronas multicuspídeas, cónicas o cuadradas, entre otras. Además, en ocasiones se observan fusiones radiculares, raíces múltiples, raíces supernumerarias, enanismo o gigantismo radicular. ${ }^{4}$

La disposición anatómica de los terceros molares mandibulares es prácticamente impredecible, ${ }^{5}$ por lo que es necesario un correcto diagnóstico para el manejo quirúrgico, prevención de complicaciones y manejo posoperatorio. El estudio radiográfico es esencial, ya que se requiere identificar el punto de menor resistencia para vencer el anclaje radicular en el hueso alveolar con las maniobras quirúrgicas. ${ }^{6}$

Los terceros molares se han clasificado radiográficamente conforme su posición respecto al segundo molar y la rama mandibular. Según Pell y Gregory, los terceros molares se pueden categorizar en nivel $A, B$ o $C$ de acuerdo con su profundidad respecto al plano oclusal y en clase I, II o III de acuerdo con el espacio disponible (respecto a la rama ascendente mandibular y el segundo molar adyacente). ${ }^{7}$ La posición espacial se ha clasificado de acuerdo con Winter en cuatro categorías de inclinación respecto al eje longitudinal del tercer molar. ${ }^{8}$ Finalmente, Sánchez Torres desarrolló una clasificación basada en la profundidad y dirección del tercer molar, número, dirección y forma de las raíces y en consideraciones complementarias como la relación con el conducto dentario inferior y la relación con el segundo molar. Esta clasificación, menos conocida que las anteriores, sin valoración con anterioridad, ${ }^{9}$ considera un mayor número de elementos.

En los ámbitos clínico, médico-legal y de investigación es importante establecer la misma percepción de un problema para diferentes observadores. En el proceso de categorización de terceros molares son básicas las clasificaciones de Pell y Gregory y Winter; la de Sánchez Torres es un método empleado en México. ${ }^{10} \mathrm{El}$ objetivo del presente estudio fue evaluar el grado de acuerdo en la valoración de terceros molares conforme a esas tres clasificaciones
(Figura 1), entre un grupo de cirujanos orales y maxilofaciales certificados y de residentes en formación.

\section{Método}

El estudio fue realizado en la Clínica de Cirugía Bucal de la Facultad de Estomatología de la Universidad Autónoma de San Luis Potosí, México. Fueron utilizadas radiografías panorámicas digitales de pacientes que acudieron a esa clínica; el estudio fue sometido a aprobación por el Comité de Ética institucional (CEI-FE-012018232072). Las radiografías fueron identificadas solo por edad y sexo del paciente a quien pertenecían. Los evaluadores fueron 10 cirujanos orales y maxilofaciales y 10 residentes de esa especialidad en el Hospital Central Ignacio Morones Prieto en San Luis Potosí, México.

Los 20 observadores registraron la clasificación de los molares mandibulares izquierdos y derechos de acuerdo con las clasificaciones de Pell y Gregory, Winter y Sánchez Torres. Cada observador evaluó 20 radiografías (10 terceros molares izquierdos y 10 derechos). Antes de la evaluación, a todos se les proporcionó un manual con las características a considerar para cada clasificación, con la finalidad de estandarizar criterios. Las radiografías fueron mostradas aleatoriamente en forma digital con suficiente nitidez y contraste. Los datos fueron consignados en hojas de recolección.

El análisis estadístico fue realizado con el programa $\mathrm{R}$ versión 3.4 , utilizando el paquete "irr". Se efectuó un análisis de concordancia mediante kappa de Fleiss para múltiples evaluadores. La significación de la prueba se determinó en $p<0.05$; la interpretación de los valores de kappa (Tabla 1) se tomó con base en la tabla reportada por Posner.11

\section{Resultados}

Los resultados del grado de concordancia se pueden observar en la Tabla 2; muestran que la clasificación con el menor grado de concordancia fue la de Pell y Gregory, mientras que la de Sánchez Torres tuvo un grado de concordancia leve para los cirujanos maxilofaciales y un grado de concordancia justo para los residentes. La clasificación de Sánchez Torres tuvo un grado de concordancia similar en su componente anatómico coronal y espacial, así como en el radicular, lo que demuestra su dominio tanto en los cirujanos maxilofaciales como en los residentes. Finalmente, la clasificación de Winter presentó el mayor grado de concordancia en ambos grupos de observadores. 


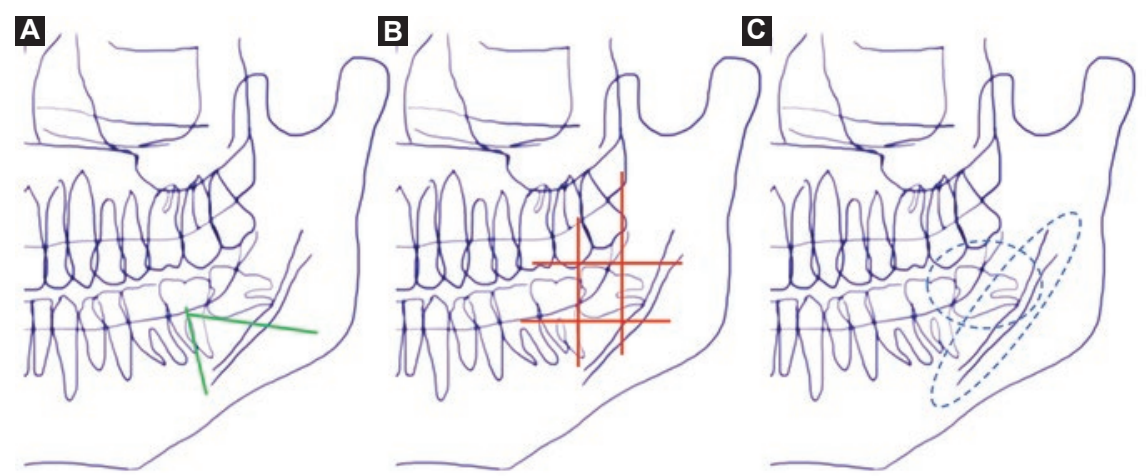

Figura 1. Representación esquemática de las clasificaciones más comunes de tercer molar mandibular. A) Clasificación de Winter, basada en la posición del tercer molar en relación con el eje longitudinal del segundo molar. B) Clasificación de Pell y Gregory, basada en la profundidad en relación con el plano oclusal del segundo molar inferior y del diámetro mesiodistal del diente retenido, conforme la distancia del segundo molar inferior y la parte anterior de la rama de la mandíbula. C) Clasificación de Sánchez Torres, basada en tres factores fundamentales: profundidad y dirección del tercer molar, número, dirección y forma de las raíces; así como en dos complementarios: relación con el conducto dentario inferior y relación con el segundo molar.

Tabla 1. Interpretación de valores de kappa

\begin{tabular}{|l|l|}
\hline Valor de kappa & Fuerza de acuerdo \\
\hline$<0.000$ & Pobre \\
\hline $0.00-0.20$ & Leve \\
\hline $0.21-0.40$ & Justa \\
\hline $0.41-0.60$ & Moderada \\
\hline $0.61-0.80$ & Sustancial \\
\hline $0.81-1.00$ & Perfecta \\
\hline
\end{tabular}

Tabla 2. Valores de kappa por grupo

\begin{tabular}{|c|c|c|c|}
\hline Grupo & Clasificación & $\begin{array}{l}\text { Kappa de } \\
\text { Fleiss }\end{array}$ & p \\
\hline CMF & Sánchez-Torres & $0.125^{\star}$ & $0.000^{* *}$ \\
\hline CMF & $\begin{array}{l}\text { Sánchez-Torres } \\
\text { (componente radicular) }\end{array}$ & $0.105^{\star}$ & $<0.001^{* *}$ \\
\hline CMF & Pell y Gregory & $0.05^{\star}$ & $0.0017^{\star *}$ \\
\hline CMF & Winter & $0.28^{\star}$ & $0.000^{\star *}$ \\
\hline Residentes & Sánchez-Torres & $0.326^{*}$ & $0.000^{\star *}$ \\
\hline Residentes & $\begin{array}{l}\text { Sánchez Torres } \\
\text { (componente radicular) }\end{array}$ & $0.338^{*}$ & $<0.001^{* *}$ \\
\hline Residentes & Pell y Gregory & $0.185^{*}$ & $0.0017^{\star *}$ \\
\hline Residentes & Winter & $0.636^{\star}$ & $0 * \star$ \\
\hline
\end{tabular}

\section{Discusión}

Existe razonable justificación de la necesidad de agrupar "objetos" con características similares para distinguir las entidades complejas de las simples. En medicina, el diagnóstico es un importante proceso de clasificación. ${ }^{12}$ Una clasificación determina la respuesta o reacción ante un objeto o grupo de objetos cuyos límites están claramente definidos..$^{12}$ Existen múltiples clasificaciones de los terceros molares, la preferencia para usar una $u$ otra recae en el criterio del cirujano y no en los parámetros numéricos de su utilidad; no existe un estándar nacional o internacional y la mayoría de las clasificaciones no han sido validadas.

Los tratamientos estomatológicos de rutina, como obturaciones, endodoncias o cirugía oral, entre otras, son seleccionados con base en la severidad, riesgos, características anatómicas y condiciones propias del paciente. En estos escenarios clínicos es fundamental clasificar la entidad patológica de forma correcta con el fin de disminuir o prevenir posibles complicaciones, así como seleccionar el tratamiento adecuado para cada paciente. Si el método de clasificación carece de la capacidad para permitir el común acuerdo entre observadores, se dificulta y entorpece el quehacer clínico.

Además de los entornos clínicos habituales, la correcta clasificación es vital para la investigación clínica. Históricamente, las clasificaciones de Winter y de Pell y Gregory han sido utilizadas para diferentes diseños de investigación en torno a cirugía de tercer molar. En la actualidad continúan usándose para definir criterios de selección, ${ }^{13}$ sin embargo, otros estudios se han enfocado en clasificar a los pacientes solo con base en el grado de impactación del tercer molar, ${ }^{14}$ el cual se relaciona con el grado de remoción ósea, sobre todo los estudios de analgesia que usan el modelo de dolor de impactación dental. ${ }^{15}$

A partir de la falta de acuerdo pueden suscitarse controversias legales, por ejemplo, la lesión al nervio alveolar inferior es una de las complicaciones que 
más se asocia con incomodidad y acciones legales por parte del paciente..$^{16}$ Por esta razón, resulta relevante determinar el grado de acuerdo de la interpretación de las clasificaciones más usadas por los cirujanos orales y maxilofaciales.

Otro aspecto en el desarrollo de nuevas escalas, instrumentos de evaluación y clasificación es que requieren un adecuado proceso de validación y confiabilidad. La validez de una prueba diagnóstica está determinada por la sensibilidad, especificidad, valor predictivo positivo y negativo de esta, que idealmente deberían tener valores mayores o iguales a $80 \%$ y que tienen la desventaja de requerir un estándar de oro, que en las clasificaciones de terceros molares no existe. ${ }^{17}$ Por su parte, la confiabilidad se refiere al grado en que se obtendrían resultados similares después de aplicar el proceso de medición más de una vez. ${ }^{18}$

En relación con la retención dental, los sistemas de clasificación actuales se basan en parámetros clíni$\cos$ y radiográficos ${ }^{16,19,20}$ para evaluar la posición de terceros molares, sin embargo, han sido aceptados sin validación previa. ${ }^{21}$ En la literatura se han encontrado diversos estudios que estiman el grado de acuerdo de las clasificaciones de Winter y de Pell y Gregory; en el presente trabajo es interesante observar el poco acuerdo en la clasificación de Pell y Gregory (Tabla 2), aceptada como estándar en la clasificación de terceros molares mandibulares. Estos resultados coinciden con los de García et al. ${ }^{17}$ y Lima et al., ${ }^{22}$ quienes demuestran que no existe fiabilidad y validez en los criterios de clasificación, ya que hay innumerables combinaciones entre las posiciones y las clases, lo que dificulta la categorización.

Desde el 2000, García et al..$^{17}$ reportaron que la clasificación de Pell y Gregory no es útil para predecir el grado de dificultad quirúrgica; adicionalmente se ha señalado que implica un conocimiento profundo de la técnica quirúrgica ${ }^{23}$ y que su bajo grado de acuerdo entre observadores está dado por su gran cantidad de categorías; ${ }^{22}$ además, gran parte de su variabilidad se debe a la interpretación subjetiva del observador para determinar qué criterio aplicar en cada caso. ${ }^{21}$

Por otro lado, los resultados en la clasificación de Winter muestran un grado de acuerdo sustancialmente más alto entre los residentes en formación (kappa $=0.636$ ) que entre los cirujanos maxilofaciales (kappa $=0.28)$, en forma similar a los reportados en 2012 por Lima et al., ${ }^{22}$ quienes indicaron un grado de acuerdo superior a $78 \%$. Se podría suponer que ese grado de acuerdo se debe principalmente a que solo se toma en cuenta una característica anatómica de los terceros molares (dirección de la pieza), en comparación con las demás variables empleadas en las clasificaciones estudiadas, hecho confirmado por Almendros Marqués et al. ${ }^{21}$

Hasta donde conocemos, no se ha reportado el grado de acuerdo de la clasificación de Sánchez Torres; esta clasificación es completa y al mismo tiempo compleja, ya que considera una gran cantidad de condiciones. A pesar de ello, a diferencia de la clasificación de Pell y Gregory, obtuvo una puntuación más alta (kappa $=0.12$ y 0.32 en los cirujanos maxilofaciales y en los residentes [Tabla 2]). Este resultado pueda deberse a que la clasificación de Sánchez Torres es más usada en México, particularmente en el centro de formación donde se desarrolló el presente estudio, aunque esto no aclara su pertinencia como posible método de clasificación.

Esta investigación presentó las siguientes limitantes: solo se incluyó un grupo de expertos formados en la misma escuela quirúrgica; a mayor número de categorías de clasificación, el valor de kappa disminuyó considerablemente, ${ }^{24}$ lo que se reflejó en baja concordancia; aunado a ello, en este tipo de estudios los observadores pueden estar concordantemente equivocados, aunque sea a niveles bajos.

Se sugiere realizar investigaciones futuras con la finalidad de validar dichas clasificaciones $y$, en lo posible, definir nuevos métodos como el propuesto por Juodzbalys y Daugela, ${ }^{25}$ basados en parámetros del entorno quirúrgico y que sigan un proceso de validación ${ }^{26}$ para confirmar su utilidad, o bien, basados en datos obtenidos de revisiones sistemáticas y metaanálisis para asegurar que sean eficientes y pertinentes tanto en la teoría como en la práctica clínica de uno de los procedimientos más frecuentes en la cirugía maxilofacial.

\section{Conclusión}

La clasificación de Pell y Gregory presentó el grado de acuerdo más bajo, seguida por la de Sánchez Torres; la de Winter demostró tener una mayor capacidad de acuerdo en cirujanos orales y maxilofaciales, así como en residentes en formación.

\section{Bibliografía}

1. Carter K, Worthington S. Predictors of third molar impaction: a systematic review and meta-analysis. J Dent Res. 2016;95:267-276.

2. Gay-Escoda C, Berini-Aytés L. Cordales incluidos: patología, clínica y tratamiento de tercer molar incluido. En: Gay-Escoda C, Berini-Aytés L. Tratado de cirugía bucal. Tomo I. España: Ediciones Ergón; 2004. 
3. Marciani RD. Third molar removal: an overview of indications, imaging evaluation, and assessment of risk. Oral Maxillofac Surg Clin North Am. 2007;19:1-13

4. Peñarrocha DM, Sanchis BJM, Sáez CU, Gay-Escoda C, Bagán SJV Escala numérica de la valoración de la dificultad quirúrica en la extracción de 190 terceros molares mandibulares incluidos. Arch Odontoestomatol. 2000;16:96-100.

5. Phillips $\mathrm{C}$, White RP. How predictable is the position of third molars over time? J Oral Maxillofac Surg. 2012;70:11-14.

6. Fuster-Torres MA, Gargallo-Albiol J, Berini-Aytes L, Gay-Escoda C. Evaluation of the indication for surgical extraction of third molars according to the oral surgeon and the primary care dentist. Experience in the Master of Oral Surgery and Implantology at Barcelona University Dental School. Med Oral Patol Oral Cir Bucal. 2008;13:E499-E504.

7. Pell GJ, Gregory GT. Impacted mandibular third molars: Classification and modified technique for removel. Dent Dig. 1933;330-338.

8. Winter G. Principles of exodontia as applied to the impacted third molar a complete treatise on the operative technic with clinical diagnoses and radiographic interpretations. EE. UU.: American Medical Book Company; 1926.

9. Sánchez-Torres J, Reyes-Fuentes R. Clasificación de terceros molares inferiores retenidos. Valoración radiográfica. Rev Estomatol. 1969:63-70.

10. Olguín-Martinez TG, Amarillas-Escobar ED. Morfología radicular de los terceros molares. Rev ADM. 2017;1:17-24.

11. Posner KL, Sampson PD, Caplan RA, Ward RJ, Cheney FW. Measuring interrater reliability among multiple raters: An example of methods for nominal data. Stat Med. 1990;9:1103-1115.

12. Jutel A. Classification, disease, and diagnosis. Perspect Biol Med. 2011; 54:189-205.

13. Talimkhani I, Jamalpour MR, Babaei H, Faradmal J. Comparison of intra-socket bupivacaine administration versus oral mefenamic acid capsule for posoperative pain management following removal of impacted mandibular third molars. J Oral Maxillofac Surg. 2019;77:1365-1370.

14. Gay-Escoda C, Hanna M, Montero A, Dietrich T, Milleri S, Giergiel E, et al. Tramadol/dexketoprofen (TRAM/DKP) compared with tramadol/paracetamol in moderate to severe acute pain: results of a randomised, double-blind, placebo and active-controlled, parallel group trial in the impacted third molar extraction pain model (DAVID study). BMJ Open. 2019;9:e023715.
15. Cooper SA, Desjardins PJ. The value of the dental impaction pain model in drug development. En: Szallasi A (editor). Analgesia: methods and protocols, methods in molecular biology. EE. UU.: Springer; 2010.

16. Maglione M, Costantinides F, Bazzocchi G. Classification of impacted mandibular third molars on cone-beam CT images. J Clin Exp Dent. 2015; 7:e224-e231.

17. García AG, Sampedro FG, Rey JG, Vila PG, Martin MS. Pell-Gregory classification is unreliable as a predictor of difficulty in extracting impacted lower third molars. Br J Oral Maxillofac Surg. 2000;38:585-587.

18. Van Stralen KJ, Stel VS, Reitsma JB, Dekker FW, Zoccali C, Jager KJ. Diagnostic methods I: sensitivity, specificity, and other measures of accuracy. Kidney Int. 2009;75:1257-1263.

19. Santosh P. Impacted mandibular third molars: review of literature and a proposal of a combined clinical and radiological classification. Ann Med Health Sci Res. 2015;5:229-234.

20. Ge J, Zheng JW, Yang C, Qian WT. Variations in the buccal-lingual alveolar bone thickness of impacted mandibular third molar: our classification and treatment perspectives. Sci Rep. 2016;6:1-9.

21. Almendros-Marqués N, Berini-Aytés L, Gay-Escoda C. Evaluation of intraexaminer and interexaminer agreement on classifying lower third molars according to the systems of Pell and Gregory and of Winter. J Oral Maxillofac Surg. 2008:66:893-899.

22. Lima CJ, Silva LC, Melo MR, Santos JA, Santos TS. Evaluation of the agreement by examiners according to classifications of third molars. Med Oral Patol Oral Cir Bucal. 2012;17:281-286

23. Yuasa $H$, Sugiura M. Clinical postoperative findings after removal of impacted mandibular third molars: Prediction of postoperative facial swelling and pain based on preoperative variables. Br J Oral Maxillofac Surg. 2004:42:209-214.

24. Lantz CA, Nebenzahl E. Behavior and interpretation of the $\kappa$ statistic: Resolution of the two paradoxes. J Clin Epidemiol. 1996;49:431-434.

25. Juodzbalys G, Daugela P. Mandibular third molar impaction: Review of literature and a proposal of a classification. J Oral Maxillofac Res. 2013;4:1-11.

26. Stacchi C, Daugela P, Berton F, Lombardi T, Andriulionis T, Perinetti G, et al. A classification for assessing surgical difficulty in the extraction of mandibular impacted third molars: description and clinical validation. Quintessence Int. 2018;49:745-753. 